\title{
Évaluation du système de traitement des déchets biomédicaux solides dans la commune de Keur Massar, en banlieue dakaroise au Sénégal.
}

\author{
Modou NDIAYE¹, Modou DIENG 2 , Ndèye Adjara NDIAYE³, Falilou Mbacké SAMBE4, Ndèye Coumba \\ KANE TOURE1 \\ 1. Laboratoire Biologie médicale, Hôpital Dalal Jamm, Golf-sud, BP : 19001, Guediawaye, Dakar, Sénégal \\ 2. Laboratoire d'Analyses et Essais (LAE), Ecole Supérieure Polytechnique, Université Cheikh Anta Diop, BP : 5085, \\ Dakar, Sénégal. \\ 3. Laboratoire de Microbiologie, Ecole Supérieure Polytechnique de Dakar, Université Cheikh Anta Diop, BP : 5085, \\ Dakar, Sénégal. \\ 4. Laboratoire d'Electrochimie et des Procédés Membranaires (LEPM), Ecole Supérieure Polytechnique, Université \\ Cheikh Anta Diop, BP : 5085, Dakar, de Dakar, Sénégal.
}

Original submitted in on $18^{\text {th }}$ November 2020. Published online at www.m.elewa.org/journals/ on $30^{\text {th }}$ April 2020 https://doi.org/10.35759/JABs.148.9

\section{RESUME}

Objectif : L'objectif général de cette étude est de diagnostiquer le système actuel de gestion des déchets biomédicaux et de proposer un système rationnel de gestion pour réduire, la charge de morbidité associée à ces types de déchets dans le district sanitaire de la commune de Keur Massar dans la banlieue dakaroise au Sénégal.

Méthodologie et résultats : Cette étude transversale et descriptive a enrôlé l'ensemble des structures et du personnel médical. Le district compte environ deux cent vingt mille quatre cent trente-trois $(220433)$ habitants en 2016 selon les estimations de l'agence nationale de la statistique et de la démographie (ANSD) pour une superficie globale de $25 \mathrm{Km}^{2}$. II dispose de onze 11 postes de santé, trois 03 maternités et un (01) centre de santé, 16 infirmiers chef de poste (ICP), des maîtresses sages-femmes et responsables d'unité de soins, 14 agents manipulant les déchets biomédicaux et 01 médecin-chef du district. Les résultats ont montré que la production de déchets mous au district sanitaire de Keur Massar est estimée à 48,04 tonnes/an, la quantité de piquants produits est estimée à 1948,287 Kg/an (1,9 tonnes/an). Les déchets d'activités de soins à risque infectieux (DASRI) représentent environ 12,48 tonnes par an. Les chefs de poste et responsables d'unité ayant reçu une formation formelle en gestion des DBM représentent $31,25 \%$ des enquêtés ; le personnel manipulant les DBM représente $21,43 \%$. Le personnel n'ayant pas reçu de formation représente $68,75 \%$ pour les ICP et responsables d'unité et $78,57 \%$ pour le personnel manipulant les déchets. Le pourcentage des chefs de poste et responsables d'unité connaissant de façon claire les risques associés aux DBM est de 62,5\%. En ce qui concerne le personnel manipulant les déchets, $42,86 \%$ connaissent les risques associés aux DBM. En ce qui concerne les moyens de prévention, $68,75 \%$ parmi les chefs d'unité affirment les avoir mis à la disposition du personnel mais $50 \%$ de ce personnel utilisent ces équipements. Chez le personnel manipulant les DBM, 57,14\% méconnaissent les risques liés aux DBM par contre chez les chefs de poste et responsables d'unité, le pourcentage est de $37,50 \%$. 
Conclusion et application des résultats : Cette étude a permis de poser un réel diagnostic de la gestion des déchets dans le district sanitaire de Keur Massar et de proposer un certain nombre de solutions qui contribueraient à une meilleure gestion des déchets biomédicaux.

Mots clés : Gestion des déchets biomédicaux, Risques infectieux, District sanitaire, Keur Massar

\section{ABSTRACT}

Objective: The overall objective of this study is to diagnose the current biomedical waste system and to propose a rational one to decrease the morbidity associated with these types of waste in the health district of Keur Massar in the suburb of Dakar Senegal.

Methodology and results: This cross sectional and descriptive study has involved all medical structures and personnel. The population of the district is about two hundred twenty thousand four hundred and thirty three (220 433) in 2016 according to the national statistics and demography agency for an overall area of $25 \mathrm{~km}^{2}$. There are 11 health posts, 03 maternity wards, and 01 health center, and 16 head nurses, midwives and care unit managers, 14 officers handling the biomedical wastes and a medical district officer. The results have shown that the production of soft waste in the medical district of Keur Massar has been estimated to 48.04 tons/year. The quantity of spikes produced is estimated to $1.948 .287 \mathrm{~kg} / \mathrm{year}$. Head post and managers who have been formally well trained in biomedical waste management (BWM) represent $31.25 \%$ of the overall entities. Biomedical waste personnel handlers represent $21.43 \%$. Those who have not been trained at all represent $68.75 \%$ among the head nurses and care waste managers, and $78.57 \%$ among the personnel handling the waste. Nurse heads and care unit managers percentage have a good understanding of all risks related to biomedical wastes is $62.5 \%$ For the personnel manipulating the wastes, $42.86 \%$ have a good knowledge of the risks related to BW (Biomedical Waste). For the prevention means, $68.75 \%$ among the health care unit managers make sure they gave these means to the personnel and $50 \%$ of this same personnel use these equipment. Concerning the biomedical waste handlers, $57.14 \%$ of the personnel have no knowledge of the risks related to biomedical waste, contrary to head nurses and unit managers where the percentage is $37.50 \%$.

Conclusion and Results Implementation: This study made it possible to establish a real diagnosis of waste management in the district of Keur Massar and propose some solutions. It has revealed a lack of means, a personnel training failure and a waste management difficulty in the district of Keur Massar. Thus, a sorting system forth with an appropriate labelling should be established in all medical structures to insure sensitization and medical personnel training as well as sorting and labelling waste managers, and to keep a waste management book and make compulsory the wearing of protection gloves and masks.

Key words: Biomedical waste management, Infectious risks, Health district, Keur Massar

\section{INTRODUCTION}

Les déchets biomédicaux (DBM) constituent un réel risque sanitaire et environnemental dans les pays en développement. Au Sénégal, malgré la volonté exprimée par les pouvoirs publics la gestion des déchets hospitaliers rencontre d'énormes difficultés. Malgré l'adoption des conventions internationales et la promulgation de lois portant code de l'environnement et de l'hygiène, la gestion des déchets biomédicaux reste préoccupante dans les pays en développement (Sall, 2008). Au Sénégal, les déchets posent de sérieux problèmes sanitaires (infections nosocomiales, etc.), environnementaux (pollutions et nuisances) et même socioculturels (ex : élimination des placentas et des membres amputés,) (PRSRSM, 2019). Diverses publications et enquêtes ont montré que les conditions actuelles d'élimination des déchets hospitaliers ne sont pas toujours satisfaisantes (Doucoure, 2004). La mauvaise gestion des déchets issus des activités de soins peut être à l'origine de maladies graves pour le personnel de santé, le personnel chargé de l'élimination des déchets, les patients et la population générale (Bop et al. 2017). 
Également, selon Mokoko et al. (2018), il y a une gestion inadéquate des déchets hospitaliers avec des effets potentiels néfastes sur la santé du personnel hospitalier et sur l'environnement. Au Sénégal, dans les structures sanitaires, il est courant de constater que les déchets biomédicaux sont collectés et entassés dans un seul dépôt, souvent dans l'enceinte de l'établissement. La manipulation des déchets biomédicaux et leur transport ne prennent pas en considération certains paramètres scientifiques tels que leur mélange avec les déchets municipaux généraux, leur dépôt sur les terrains en plein air et leur fouille sans précaution dans des décharges sauvages et sont fréquemment visités par les enfants, les chiffonniers et les animaux. Le manque d'infrastructures, le faible niveau d'éducation font que l'hygiène hospitalière reste un problème de santé publique avec ses conséquences qui sont: les risques infectieux, la pollution atmosphérique, la dégradation de l'environnement par l'enfouissement et les risques chimiques par la manipulation de produits dangereux. L'élimination sans précaution des déchets d'activité de soins est une source de danger pour la santé des populations; les aiguilles et les seringues contaminés représentent un risque particulier, car si elles ne sont pas éliminées correctement, elles

\footnotetext{
METHODOLOGIE

Données socio-démographiques du district de Keur Massar : Le district sanitaire de Keur Massar se trouve dans la commune d'arrondissement de Keur Massar, précisément dans le département de Pikine, dans la région de Dakar. Suite à la loi sur la régionalisation de 1996, la commune d'arrondissement de Keur Massar fût créée ; elle compte en 2016 deux cent vingt mille quatre cent trente-trois (220 433) habitants, soit 17,22\% de la population départementale avec une densité de 8817,32 habitants/Km² (MEFP/ANSD, 2015) par comparaison à la moyenne nationale qui est de 71,2 habitants $/ \mathrm{Km}^{2}$. Sur le plan sanitaire, le district compte un (01) centre de santé de niveau 1, construit en 2010, onze (11) postes de santé publics, trois (03) maternités. La capacité d'accueil du district se résume en moyenne au nombre de 122 lits disponibles au niveau du centre de santé, des postes de santé et des maternités enquêtés.
}

risquent d'être remises dans des emballages et recyclées donnant lieu à une réutilisation dangereuse. Les résultats de l'OMS en 2003, dans 22 pays en développement ont montré que la proportion des établissements de santé qui ne traitent pas les déchets de soins est de 18 à $64 \%$. Cette étude montre qu'en plus des risques pour la santé publique, en l'absence d'une bonne gestion, le risque d'être infecté par le VHB, le VHC et le $\mathrm{VIH}$ après piqûre accidentelle avec une aiguille utilisée pour le patient infecté est respectivement de $30 \%, 1,8 \%$ et $0,3 \%$ (Dieu, 2010). Au Sénégal les déchets biomédicaux posent un sérieux problème car ils constituent un risque certain pour la santé des populations et pour l'environnement. En effet le Sénégal produit annuellement 1424 tonnes de déchets biomédicaux, provenant essentiellement des structures hospitalières de la région de Dakar. Les déchets infectieux représentent $68 \%$ de l'ensemble des déchets produits par les structures sanitaires contre $27 \%$ de déchets généraux (ONU-Habitat, 2004). Le but de ce travail est de décrire la gestion des déchets biomédicaux au sein des structures sanitaires dans la commune de Keur Massar, dans la banlieue dakaroise, et de contribuer à l'amélioration de leur mode de traitement.

Méthodologie de l'étude : L'objectif général de cette étude est de proposer un système rationnel de gestion durable des déchets biomédicaux dans le district sanitaire de Keur Massar afin de réduire la charge de morbidité associée à ces types de déchets. Cet objectif général est décliné en cinq objectifs spécifiques. II s'agit :

d'apprécier le niveau de connaissance du personnel en matière de gestion des DBM ;

d'estimer la quantité de DBM produite dans le district ; de décrire les techniques actuelles de gestion des DBM dans le district ;

d'élaborer un plan de gestion appropriée des DBM avec coût et financement ;

de formuler des recommandations pour un système de gestion durable.

II s'agit d'une étude transversale descriptive qui s'est étalée sur une période de trois mois allant du 01 
septembre au 30 novembre 2016 couvrant l'ensemble des structures sanitaires publiques du district notamment les postes de santé, les maternités et le centre de santé.

Modalités de l'enquête : Dans le cadre de cette étude, II a été conçu trois (03) types de questionnaires avec des critères de performances; un (01) à l'attention du médecin-chef du district axé sur le management de la gestion des DBM, un (01) à l'attention des responsables d'unité, des infirmiers chefs de poste (ICP) et des maîtresses sages-femmes et un (01) autre à l'attention du personnel manipulant les DBM.

Echantillonnage : L'échantillonnage a été effectué au niveau de huit (08) postes de santé, deux (02) maternités et le centre de santé avec ses différentes unités de manière exhaustive. Ainsi nous avons enquêté seize (16) infirmiers chefs de poste, maîtresses sages-femmes et responsables d'unité, quatorze (14) agents impliqués dans la manipulation des déchets et le médecin-chef du district. Tout le personnel technique étant inclus d'office. Les motifs de l'enquête et la démarche méthodologique leur sont oralement exposés. L'anonymat des enquêtés est respecté et leur approbation ainsi que celle du chef de district sont requises avant la poursuite des opérations. Les agents qui n'ont pas été enrôlés dans l'étude sont: les agents de santé refusant de se soumettre à l'enquête, les agents qui n'ont pas correctement répondu au questionnaire et les agents qui sont dans des unités ne produisant pas de DBM.

Collecte des données : Nous avons utilisé comme techniques de collecte, des entretiens individuels, des questionnaires et des observations. Cette collecte a été faite grâce à des outils de collecte conçus à cet effet à savoir des questionnaires. En ce qui concerne la quantification et la caractérisation des déchets au niveau du district, un calcul sur la base des ratios " production/lit " en fonction de la catégorie de la structure et de la production/patient traité en ambulatoire sera utilisé.

Quantification des déchets produits: Pour quantifier, nous avons utilisé la méthode du ratio de production journalière par lit et du ratio de production par patient traité en ambulatoire. Un certain nombre d'études ayant été réalisées sur le sujet, dans notre pays, ou dans d'autres régions du monde ont permis d'établir une formule pour quantifier les DBM en fonction du type d'établissement sanitaire.

\section{Gisement des hospitalisations/jour $=$ Ratio production journalière/lit $\mathrm{x}$ Nombre de lits $\mathrm{x}$ Taux d'Occupation Moyen (TOM) Gisement en ambulatoire/jour $=$ Ratio de production/patient $\mathbf{x}$ Nombre de patients/jour}

La somme de ces deux produits donne la production journalière moyenne de la structure. Sur la base de mesures expérimentales effectuées dans plusieurs structures par l'Institut Africain de Gestion Urbaine

\section{RESULTATS}

Quantification des déchets produits : La production journalière de déchets mous au district sanitaire de Keur Massar est estimée à 131,64 Kg, soit 48 048,6 $\mathrm{Kg} / \mathrm{an}$, ce qui équivaut en moyenne à 48,04 tonnes/an. En considérant les déchets piquants issus des services de vaccination et du laboratoire du centre de santé, services qui génèrent la quantité la plus importante des objets contondants, nous pouvons les estimer respectivement à un nombre d'aiguilles de 168722 et 8395 soit 177117 aiguilles par an. Sachant qu'une aiguille pèse en moyenne $11 \mathrm{~g}$, la quantité d'aiguilles produite est de 1948,287 Kg/an (1,9 tonnes/an). La production totale est ainsi estimée à 49,94 tonnes/an. Etant donné que les déchets d'activités de soins à risque infectieux (DASRI) représentent environ 10 à 25 $\%$ de l'ensemble des déchets produits, nous pouvons
(IAGU) (Mbengue M.F. 1999), nous utilisons les ratios suivants : Hôpitaux $=0,26 \mathrm{~kg} /$ /it/jour ; Centre de santé = $0,37 \mathrm{~kg} / \mathrm{it}$ /jour ; Poste de santé $=0,2 \mathrm{~kg} /$ patient/jour.

quantifier ces DASRI en considérant le pourcentage le plus élevé c'est-à-dire $25 \%$ pour maximiser. La Quantité de DASRI correspond alors à : 49,94*0,25 c'est-à-dire à 12,48 tonnes par an (Tableau 1).

Formation du personnel en gestion des DBM : Les résultats de l'enquête ont également montré que certaines personnes interrogées ont suivi une formation en gestion des déchets biomédicaux tandis que d'autres n'ont pas bénéficié d'une telle formation. Ainsi, les chefs de poste et responsables d'unité ayant reçu une formation formelle en gestion des DBM sont au nombre de cinq (05), soit $31,25 \%$ du total des enquêtés; le taux est plus faible chez le personnel manipulant les DBM $(21,43 \%)$, c'est-à-dire trois (03) des quatorze qui ont été enquêtés ont reçu une formation datant de plus de deux (02) ans. Le 
personnel n'ayant pas reçu de formation représente $68,75 \%$ pour les Infirmiers Chef de Poste ICP et responsables d'unité, $78,57 \%$ pour le personnel manipulant les déchets. Le médecin-chef quant à lui, a reçu une formation et affirme que des sensibilisations en gestion des déchets biomédicaux sont effectuées une fois par mois, de même que des supervisions et contrôles sont effectués. Le score moyen obtenu par chaque cible enquêtée en matière de formation en gestion des DBM est consigné dans le tableau 2. Ce tableau montre qu'il y'a une déficience notoire en matière de formation et de sensibilisation en gestion des déchets et ce manque de formation est plus élevé chez le personnel manipulant les DBM 78,57\% que chez les chefs de poste et responsables d'unité avec un pourcentage de $68,75 \%$.

\section{Tableau 2 : Répartition du score du personnel en fonction de la formation en gestion des} DBM.

\begin{tabular}{cccc|} 
Personnel enquêté & Effectif & Formés & Non formés \\
\hline Chefs de poste et responsables d'unité & 16 & $\mathbf{3 1 , 2 5} \%$ & $\mathbf{6 8 , 7 5} \%$ \\
Personnel manipulant les DBM & 14 & $\mathbf{2 1 , 4 3 \%}$ & $\mathbf{7 8 , 5 7 \%}$ \\
Médecin-chef & 01 & $100 \%$ & $0 \%$ \\
\hline
\end{tabular}

Connaissance des risques associés aux DBM et les mesures de sécurité à prendre : L'étude a également montré le pourcentage du personnel connaissant les risques liés aux déchets biomédicaux et les mesures de prévention qui doivent être prises. Les pourcentages du personnel connaissant les risques liés aux déchets biomédicaux et les mesures de prévention qui doivent être prises sont consignés dans le tableau 3.

Tableau 3 : Répartition du score du personnel selon leur connaissance des risques liés aux DBM et les moyens de prévention utilisés

$\begin{array}{lccc}\text { Personnel enquêté } & \text { Effectif } & \begin{array}{c}\text { Connaissance des } \\ \text { Risques de maladie }\end{array} & \begin{array}{c}\text { Connaissances des } \\ \text { Moyens de prévention }\end{array} \\ \begin{array}{l}\text { Chefs de poste et } \\ \text { responsables d'unité }\end{array} & 16 & 62,5 \% & 68,75 \% \\ \begin{array}{l}\text { Personnel } \\ \text { manipulant les DBM }\end{array} & 14 & 42,86 \% & 50 \%\end{array}$

Ainsi, parmi les seize chefs de poste et responsables d'unité, dix (10), soit $62,5 \%$ connaissent de façon claire les risques associés aux DBM et ont pu citer trois types de maladies qui peuvent découler de la blessure avec les objets contondants; en ce qui concerne les moyens de prévention mis à la disposition du personnel manipulant les déchets, onze (11), soit $68,75 \%$ affirment qu'ils mettent à la disposition de ce personnel des EPI (gants, masque, blouse). En ce qui concerne le personnel manipulant les déchets, six (06), soit 42,86 $\%$ connaissent les risques associés aux DBM et sept (07) confirment qu'ils utilisent les EPI, soit $50 \%$. En ce qui concerne les cas d'accidents exposant au sang (AES) recensés durant les douze derniers mois, trois chefs de poste sur les seize $(18,75 \%)$ affirment avoir recensé des cas avec un taux supérieur à $0,5 \%$ mais malheureusement aucun protocole formalisant la conduite à tenir n'a été mis en place. Par contre six responsables sur les seize $(37,5 \%)$ ont formalisé un protocole de conduite à tenir en cas d'AES ; deux (02) des responsables, soit $12,5 \%$ confirment que le personnel a été vacciné contre le tétanos et l'hépatite B. Chez le personnel manipulant les DBM enquêté, nous avons noté un pourcentage très élevé de cas d'AES car $42,85 \%$ affirment avoir subi un AES au cours des douze derniers mois et $50 \%$ de ce personnel ayant subi un AES ne disposent pas d'un protocole écrit de conduite à tenir. Seuls $21,42 \%$ du personnel manipulant les DBM disposent d'un tel protocole. Pour la prévention contre certaines infections comme I'hépatite $B$ et le tétanos, seuls $35,71 \%$ ont été vaccinés. D'après les données de l'enquête, les chefs de poste et responsables d'unité qui n'ont pas mis à la disposition du personnel au moins trois types d'EPI 
représentent $31,25 \%$. Cependant le personnel manipulant les déchets et ne disposant pas de ces

\section{DISCUSSION}

Le travail montre que les différentes catégories de déchets produits sont: les déchets assimilés aux ordures ménagères (DAOM), les déchets piquants et tranchants, les déchets d'Activités de Soins à Risque Infectieux (DASRI), les déchets anatomiques, les déchets pharmaceutiques et les films de radiographie produits exclusivement au service de radiologie. Le tri types d'EPI (gants, masques, tenue, bottes) représente $50 \%$ des enquêtés.

des déchets est inadapté. II n'y a pas de séparation des DAOM et des DASRI. Le seul système de tri qui existe est celui des piquants/tranchants car ces derniers sont recueillis dans des boîtes de sécurité destinées à cet effet. Pour le conditionnement des déchets, nous avons constaté qu'il n'y a pas de système de codage par couleur des poubelles. (Figure 1).

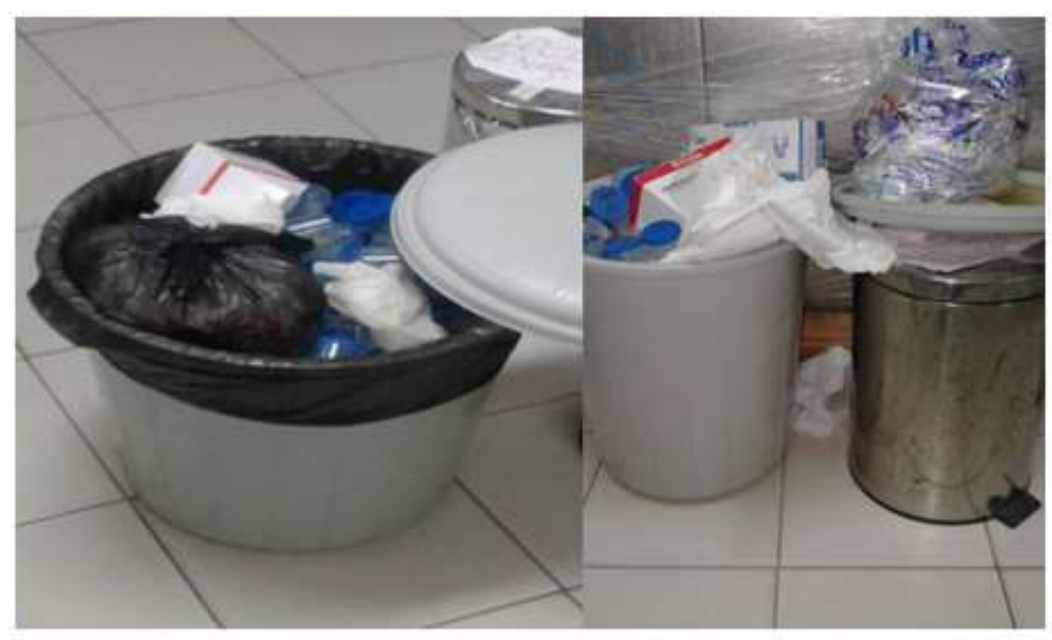

Figure 1 : Conditionnement des déchets (Cliché M. NDIAYE 2016)

La collecte et le ramassage des déchets doivent se faire de façon quotidienne mais dans certains services la personne chargée d'acheminer ces déchets passe tous les deux jours, c'est le cas du laboratoire. Les déchets liquides sont directement déversés dans les éviers ou les égouts sans traitement au préalable ce qui peut entrainer de graves conséquences environnementales. Le transport des DBM est interne et se fait manuellement du lieu de production jusqu'au lieu de stockage lorsque la quantité produite n'est pas importante, dans le cas contraire, des chariots sont utilisés. Pour amener les sacs contenant les déchets à l'endroit du brûlage, le transport se fait manuellement.
Les boîtes de sécurité sont transportées à l'aide de chariot roulant par les mêmes techniciens de surface qui assurent la collecte quotidienne; ils sont dotés d'équipements de protection individuelle (botte, gant, parfois masque). Au niveau, aussi bien, des postes de santé visités qu'au centre de santé, il n'y a pas de zone de stockage appropriée. Au centre de santé, les déchets biomédicaux sont dépotés à côté de la maternité tous les jours après ramassage, les déchets assimilés aux ordures ménagères sont aussi stockés dans le même endroit et seront éliminés par un groupement d'intérêt économique (GIE) à la déchèterie municipale (Figure 2). 


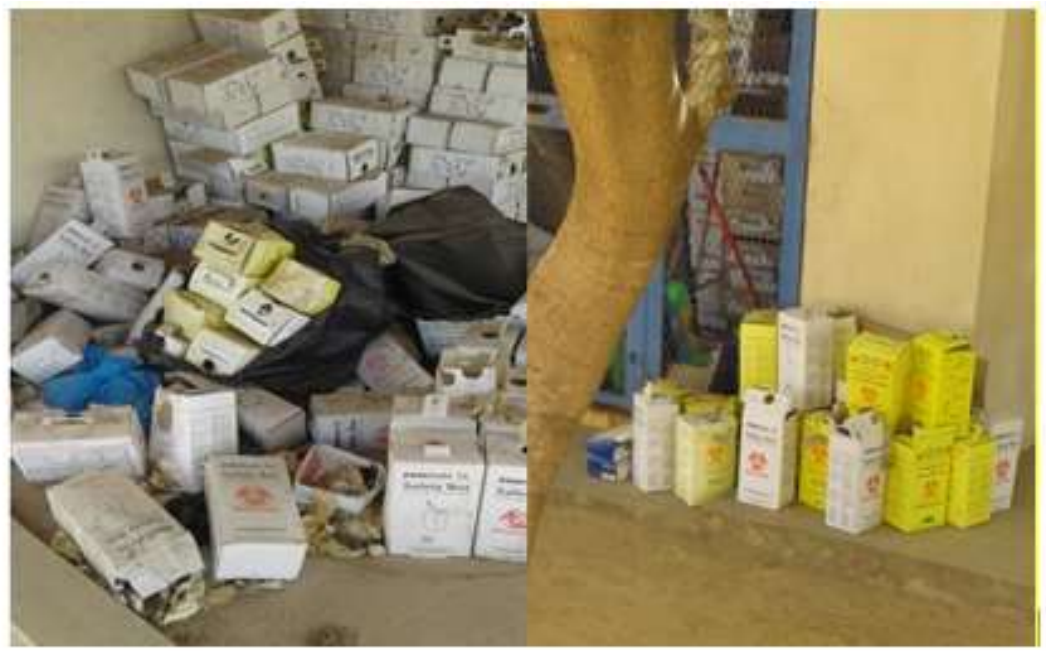

Figure 2 : Stockage des boîtes de sécurité (Cliché M. NDIAYE 2016)

Aucun traitement n'est fait au préalable avant la destruction des déchets surtout les déchets liquides qui sont directement éliminés dans les égouts ou versés dans les éviers. Du fait de la panne de l'incinérateur, les autres déchets sont brûlés à l'air libre avec toutes les conséquences que cela peut engendrer (pollution de l'environnement). Dans les postes visités, les déchets ne sont même pas brûlés mais ramassés par les camions et directement jetés au dépotoir public de Mbeubeuss dans la banlieue dakaroise. Au centre de santé, les déchets sont éliminés par brûlage (figure 3).

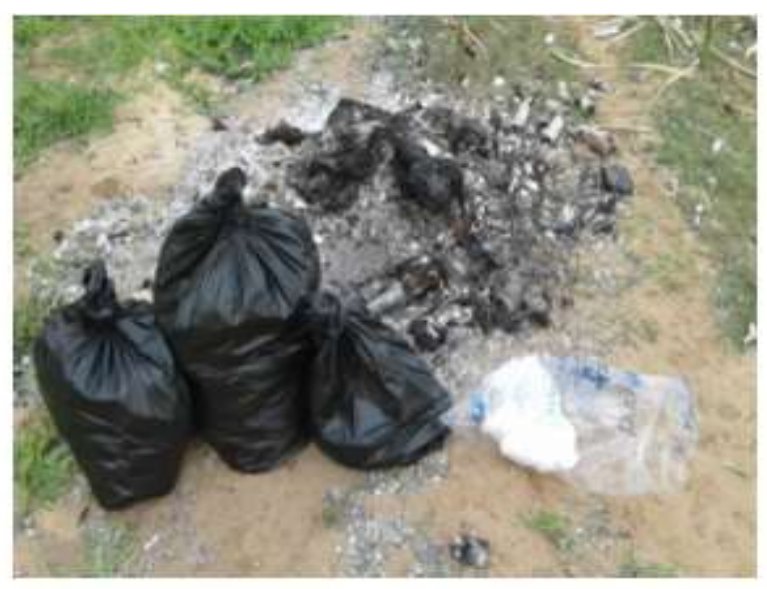

\section{Figure 3 : Lieu d'élimination par brûlage des déchets \\ (Cliché M. NDLAYE 2016)}

La mauvaise gestion des déchets biomédicaux constitue un véritable problème de santé publique. L'ampleur de ce problème est une préoccupation aussi bien pour les pays développés que pour les pays en développement. Le problème est d'autant plus grave que toutes les personnes fréquentant les structures sanitaires sont exposées aux risques de contamination. Les plus exposés sont les malades, le personnel de santé et particulièrement les préposés à la manipulation des déchets. Dans cette étude, les sacs poubelles utilisés ne répondent pas aux normes car ils ne sont pas autoclavages. Par contre les objets contondants sont recueillis dans des conteneurs qui leur sont destinés mais ces derniers sont en carton et peuvent être détruits lorsque les conditions de stockage ne répondent pas aux normes. Une étude de Bop et al. (2017) réalisée à Goudiry au Sénégal a révélé qu'en dehors des seringues et des aiguilles, il n'existait aucun 
système de conditionnement des déchets dans les structures de santé. Le remplissage des boîtes de sécurité n'était pas conforme aux normes. Les poubelles ne disposaient pas de couvercle et n'étaient pas nettoyées après le déversement des déchets. Les boîtes de sécurité sont pleinement remplies au lieu d'être remplies au $2 / 3$ de leur volume. Selon une étude similaire (Mokoko et al 2018), la mauvaise gestion des déchets hospitaliers et du matériel biomédical constitue un véritable problème de santé par son ampleur, sa gravité et sa vulnérabilité. Dans les postes de santé, les déchets ne sont même pas brûlés contrairement au centre de santé où les déchets sont éliminés par brûlage. Cela a été décrit par une étude menée par

\section{CONCLUSION ET APPLICATION DES RÉSULTATS}

La gestion des déchets biomédicaux dans les structures sanitaires pose une véritable problématique. Cependant les risques pour la communauté et les risques de pollution de l'environnement demeurent toujours du fait des manquements notés dans le système qui est mis en place. Dans le district de Keur Massar la plupart des déchets provenant des postes de santé sont dépotés à la décharge de Mbeubeus, lieu où l'on retrouve une importante population de chiffonniers et de recycleurs. Dans son ensemble, le système de gestion des DBM dans le district souffre et nécessite l'apport de solutions pour assurer un tri adéquat, un transport sécurisé et un traitement efficace. L'étude a révélé un manque de moyens logistiques, une défaillance sur la formation du personnel et une difficulté de gestion des déchets dans la commune de Keur Massar. Ainsi, il convient d'établir un système de tri avec un étiquetage approprié dans tous les

\section{REFERENCES}

A. m. d. I. s. d., Atlas mondial des personnels de santé. 2009. Organisation mondiale de la santé, OMS, Densité de personnel sanitaire.

Bop M.C., Sow P.G., Gueye B., Tall A.B., Ka O. et Diop E.M. 2017. Etude de la gestion des déchets biomédicaux dans le district sanitaire de Goudiry au Sénégal. Mali medical 2017 tome XXXII $n^{\circ} 3$.

Dieu N.J.D., 2010. Connaissances, attitudes et pratiques des professionnels de santé sur la gestion des déchets hospitaliers. Mémoire de Licence en science de la santé.

Doucouré D., 2004. Plan National de Gestion des déchets biomédicaux, p. 91.
Ndiaye, (2012) où il a souligné que l'incinération était le mode d'élimination le plus utilisé dans quatre structures hospitalières sur cinq au Sénégal. En 2002, une étude réalisée par Nianga a montré que l'incinération avec un taux de $30,48 \%$ est le moyen le plus utilisé même si elle constitue un important émetteur de polluants atmosphériques. L'étude révèle également que les déchets piquants issus des services de vaccination et du laboratoire du centre de santé, sont estimés à 1,9 tonnes/an. Dans une étude semblable (Mokoko, 2018), les principaux déchets tranchants rencontrés dans les services du CHU-B sont des seringues, lames et aiguilles avec des taux respectifs de 83,33\%, 82,05 \%, $91,02 \%$.

établissements sanitaires, d'assurer la sensibilisation et la formation du personnel médical et des gestionnaires des déchets au tri et au marquage des déchets, de tenir un registre de gestion des déchets et d'exiger le port obligatoire de blouses, de gants de protection et de masques. Pour une meilleure gestion des déchets, il est essentiel d'impliquer la collectivité locale, renforcer la sensibilisation, promouvoir la collaboration et le partenariat avec les organismes financiers. Cette approche contribuera à améliorer de manière significative les pratiques quotidiennes de gestion des déchets biomédicaux tout en mettant en place des stratégies pour assurer une élimination sûre et correcte de ces déchets dans tout le district. Au regard de ces différentes pratiques sur le système de gestion des déchets dans le district sanitaire de Keur Massar, il urge de mettre en place un système de gestion des déchets performant et durable.

Mbengue M.F. 1999. Déchets biomédicaux en Afrique de l'Ouest : problèmes de gestion et esquisse de solution, IAGU-PGU, pp 13-27.

MEFPIANSD. 2015. Projection de la population du Sénégal 2013-2025. Agence Nationale de la Statistique et de la Démographie (ANSD).

Mokoko J.B., Atipo I.B., Mimiesse J.F., lloukou P.J. et Takale R., 2018. Gestion des Déchets Hospitaliers et du Matériel Biomédical au CHU de Brazzaville. Health Sci. Dis : Vol 19 (2).

Ndiaye M., Metghari L., Soumah M.M. et Sow M.L., 2012. Gestion des déchets biomédicaux au sein de cinq structures hospitalières de Dakar, Sénégal. Bull. Soc. Pathol. Exot, 105:296-304. 
Ndiaye et al., J. Appl. Biosci. 2020 Évaluation du système de traitement des déchets biomédicaux solides dans la commune de Keur Massar, en banlieue dakaroise au Sénégal.

Nianga B., 2002. Gestion Des déchets biomédicaux au CHUB. Mémoire de licence en santé Publique. Université Marien NGOUABI de BrazzavilleCongo ; 2002:32.

Onu-habitat,. 2004. Profil national du Sénégal.

PRSRSM, Programme de renforcement des systèmes régionaux de surveillance de maladies, 2019.
Réactualisation du plan de gestion des déchets biomédicaux au Sénégal.

Sall A. T., 2008. Etude évaluative de la gestion des déchets issus des activités de vaccination dans le district sanitaire de Mbao à Dakar. Mémoire diplôme interuniversitaire à IRSPOuidah Bénin. 